\title{
Typologie des Brackwassers
}

\author{
Cornelis den Hartog
}

\author{
Rijksherbarium, Leiden, Niederlande
}

\begin{abstract}
Typology of brackish water. The Venice System for classification of brackish waters is based on one single factor, salinity, and consequently is not fit for a biological classification. I have amended the system by defining the different subdivisions according to their biological features (DEN HARTOG 1960). It appears that the transition area between fresh and sea water usually shows a tripartition. In the different types of brackish water this tripartition is caused by different factors connected with salinity. In the estuaries the enormous daily salinity fluctuations exercise a too great pressure on the osmotic capacity of many species and thus form an effective barrier for them. In the Baltic Sea, where the salinity conditions are more or less stable, the salinity limit for many species is also the limit of their salinity tolerance. In lagoons and isolated brackish waters the minimum and maximum salinities are decisive for the absence or presence of several species. Although in all these waters the subdivision in an oligohaline, a mesohaline and a polyhaline subtype is evident, there is no reason to suppose that the ranges of the corresponding subtypes will coincide, the more so as their boundaries are determined by different environmental factors. In this paper, therefore, a typological system for the brackish waters is proposed, based on the continuity or discontinuity of the transition between fresh and sea water, and on the periodicity of the salinity fluctuations. In some of the eight recognized types a subdivision according to the amended Venice System is possible.
\end{abstract}

\section{EINLEITUNG}

Während des Symposions für die Klassifizierung der Brackwässer in Venedig im Jahre 1958 wurde bei den Teilnehmern Ubereinstimmung über das sogenannte „Venice System " erzielt, dessen Einteilung auf dem einzigen Merkmal des mittleren Salzgehaltes des Wassers basiert. Dieses System wurde für eine Universalanwendung empfohlen.

Wenn die Einteilung des Brackwassers an erster Stelle eine Klassifizierung der dort existierenden Biozönosen enthalten soll, ist es verständlich, daß eine Einteilung nach dem mittleren Salzgehalt niemals genügen kann, sondern daß es notwendig ist, die Klassen des Systems anhand der Flora und Fauna zu definieren, also mit biologischen Daten. Nachher kann man das auf biologischen Tatsachen aufgebaute Schema mit den Faktoren des abiotischen Milieus korrelieren, und ich bin ganz damit einverstanden, daß die Salinität wohl der erste Faktor ist, welchen man für diesen Zweck wählen sollte. Zweifelsohne liegt solch eine Korrelation auch dem "Venice System" zugrunde, obgleich das nicht in der "Final Resolution" (1959) des Symposions gesagt wird. Wie anders konnten Schwierigkeiten bei der Abgrenzung der verschiedenen Klassen entstehen? (Segerstråle 1959, Petit \& 
SCHACHTER 1959, D’AnCona 1959, u. a.) In dem "Venice System“ werden jedoch nicht die biologischen Erscheinungen, sondern die Salzgehaltsangaben und wohl besonders die mittlere Salinität berücksichtigt. Man war sich aber bei der Aufstellung des Systems sehr wohl bewußt, daß allerlei andere Faktoren, wie die Salzgehaltsschwankungen, die Maximal- und Minimalsalzwerte, die Temperaturzyklen, usw. auch eine wichtige Rolle spielen, wenn man die Salinitätsstufen mit den biologischen Verhältnissen im Einklang zu bringen wünscht, doch man konnte das im System selber nicht zum Ausdruck bringen. In dieser Hinsicht weicht das "Venice System" nicht grundsätzlich ab von älteren Systemen der Brackwasserbiologen REDEKE (1933) und VäLIKANGAS (1933), und ich betrachte deshalb die ambitiöse Empfehlung für die Universalanwendung der neuen Einteilung als eine Überschätzung der wirklichen Bedeutung des in Venedig übereingekommenen Systems.

Um aus dieser Sackgasse herauszulkommen, habe ich das "Venice System" erweitert durch die Hinzufügung einer kurzen Charakteristik der biologischen Erscheinungen, welche in den verschiedenen Salinitätsklassen des Systems auftreten (DEN HARToG 1960). Hierdurch hat die Einteilung eine biologische Basis bekommen. Die Salinitätsdaten geben jetzt annähernd an, unter welchen Umständen die beschriebenen Erscheinungen normaliter realisiert werden. Sie sind nicht länger die Einteilungskriterien für das System. Obwohl das amendierte „Venice System“ nun erlöst ist von den klemmenden Bändern der Salinitätswerte und folglich viel flexibler geworden ist, bieten sich in der Natur oft Situationen dar, vor welchen das System vollkommen versagt.

Nun bin ich einige Jahre in der glücklichen Lage gewesen, das Brackwasser des südwest-niederländischen Deltagebietes eingehend untersuchen zu können. Ganz besonders habe ich mich mit einem Studium der faunistischen Gliederung in den Aestuaren der Flüsse Rhein, Maas und Schelde (DEN HARTOG 1961) beschäftigt. Daneben habe ich die Benthosgemeinschaften und den Salzzykius in vielen, innerhalb der Deiche gelegene Brackwässer während einiger Jahre beobachten können. Als eine Folge dieser Untersuchungen sind mir einige Gedanken gekommen, welche vielleicht für eine den natürlichen Verhältnissen besser angepaßte Einteilung des Bradkwassers von Bedeutung sein können.

\section{SALZGEHALTSGRENZE VON ARTEN IN AESTUAREN UND IN ANDEREN BRACKWASSERTYPEN}

Die Flußmündungen im südwestlichen Teil der Niederlande zeigen eine deutliche Differenzierung in der Flora und Fatna, welche mit dem mittleren Salzgehalt in engem Zusammenhang steht. Die Übergangszone von Süß- nach Meerwasser zeigt eine charakteristische Dreiteilung:

1. Ein Oligohalinikum, gekennzeichnet durch einige euryhaline Süßwasserformen, wenige euryhaline Vertreter des marinen Elements und eine kleine Anzahl Brackwasserarten; mittlerer Salzgehalt: 0,3 bis $1,8 \% \mathrm{Cl}^{\prime}$.

2. Ein Mesohalinikum, charakterisiert durch zahlreiche Brackwasserorganismen, nur wenige euryhaline marine Arten und praktisch keine Süßwasserrepräsentanten; mittlere Salinität: 1,8 bis etwa $10 \% \mathrm{Cl}^{\prime}$. 
Tabelle 1

Niedrigste mittlere Salinitätswerte in $\% \mathrm{Cl}^{\prime}$ von einigen euryhalinen marinen Organismen in den niederländischen Aestuaren und der Ostsee

\begin{tabular}{|c|c|c|}
\hline & Aestuar & Ostsee \\
\hline $\begin{array}{l}\text { Potamogetonaceae: } \\
\text { Zostera marina L. }\end{array}$ & 14 & 3 \\
\hline $\begin{array}{l}\text { Chlorophyceae: } \\
\text { Cladopbora rupestris (L.) KüTz. } \\
\text { Blidingia minima (NÄG.) KYLIN }\end{array}$ & $\begin{array}{c}14 \\
\text { bis ins Süßßwasser }\end{array}$ & $<3$ \\
\hline $\begin{array}{l}\text { Phaeophyceae: } \\
\text { Fucus vesiculosus L. } \\
\text { Elachista fucicola (VeldeY) AResch. } \\
\text { Spbacelaria plumigera HoLmes } \\
\text { Sphacelaria britannica SAUv. } \\
\text { Chorda filum (L.) STACKH. }\end{array}$ & $\begin{array}{l}2 \\
14 \\
16,5 \\
16,5 \\
14\end{array}$ & $\begin{array}{l}2 \\
2 \\
3 \\
3 \\
1,6\end{array}$ \\
\hline $\begin{array}{l}\text { Rhodophyceae: } \\
\text { Bangia fuscopurpurea (DILLW.) LrNGB. } \\
\text { Rhodochorton purpureum (LIGHTF.) RosENV. } \\
\text { Pbyllopbora membranifolia (GoOD. \& WOODW.) J. AG. } \\
\text { Hildenbrandtia prototypus NARDO } \\
\text { Polysipbonia nigrescens (DILLW.) GREv. } \\
\text { Callithamnion roseum (ROTH) LYNGB. }\end{array}$ & $\begin{array}{c}\text { bis ins Süßwasser } \\
14 \\
14 \\
2 \\
14 \\
7\end{array}$ & $\begin{array}{r}<1 \\
<1 \\
3 \\
<1 \\
2 \\
3\end{array}$ \\
\hline $\begin{array}{l}\text { Crustacea: } \\
\text { Gammarus locusta (L.) } \\
\text { Melita palmata (MONT.) } \\
\text { Calliopius laeviusculus (KRøYER) } \\
\text { Idoted granulosa RATHKE }\end{array}$ & $\begin{array}{l}10 \\
7 \\
16,5 \\
15\end{array}$ & $\begin{array}{l}3 \\
4 \\
3,4 \\
3,2\end{array}$ \\
\hline $\begin{array}{l}\text { Polychaeta: } \\
\quad \text { Nereis diversicolor O. F. MüLLER }\end{array}$ & bis ins $S \ddot{ } ß ß w a s s e r$ & 2,7 \\
\hline $\begin{array}{l}\text { Turbellaria: } \\
\text { Archilopsis unipunctata (FABR.) } \\
\text { Coelogynopord schulzii MEIXNER } \\
\text { Monocelis lineatal (O. F. MÜLLER) } \\
\text { Promonotus schultzei MEIXNER } \\
\text { Provortex karlingi Ax } \\
\text { Pseudograffilla arenicola MEIXNER } \\
\text { Proxenetes flabellifer JENSEN } \\
\text { Promesostoma marmoratum (SCHULTZE) } \\
\text { Acrorbynchides robustus (KARLING) }\end{array}$ & $\begin{array}{c}0,3 \\
10 \\
11 \\
11 \\
3 \\
3 \\
3 \\
3 \\
3\end{array}$ & $\begin{array}{l}0,6-1 \\
3 \\
3 \\
3 \\
3 \\
3 \\
3 \\
2,5 \\
3\end{array}$ \\
\hline $\begin{array}{l}\text { Mollusca: } \\
\text { Mytilus edulis L. } \\
\text { Macoma baltbica(L.) }\end{array}$ & $\begin{array}{c}7-8 \\
3\end{array}$ & $\begin{array}{l}2,2 \\
1,4\end{array}$ \\
\hline
\end{tabular}

3. Ein Polyhalinikum, mit Biozönosen, die hauptsächlich aus euryhalinen marinen Organismen und nur sehr wenigen Brackwasserarten bestehen; mittlerer Salzgehalt: etwa 10 bis $16,5 \% \mathrm{Cl}^{\prime}$.

Die genannte Dreiteilung ist nicht nur korreliert mit dem mittleren Salzgehalt, sondern die Grenzen der Bezirke sind zugleich die Ubergänge von Salzgehaltsschwankungsgebieten. Das Mesohalinikum zeigt die bedeutendsten Salinitätsfluktuationen, also die markanteste Salzgehaltsstratifkation. Im Oligohalinikum und Polyhalinikum sind die Abweichungen vom mittleren Zustand viel geringer. Die Ubergänge zwischen 
Mesohalinikum und Oligohalinikum und zwischen Polyhalinikum und Mesohalinikum sind oft ziemlich diskontinuierliche Sprunggebiete. In der Mündung des Haringvliets maß ich einmal auf beiden Seiten einer Schaumlinie Salzgehalte von 12,28 und $5,26 \% \mathrm{Cl}^{\prime}$ (29. Juni 1961). Das wenig ausgedehnte Mesohalinikum der Volkerak ist tatsächlich ein großes Sprunggebiet zwischen Oligohalinikum und Polyhalinikum.

Feinere Unterteilungen, wie sie CASPERs (1959) für die Elbe angibt, wurden nicht beobachtet, was ohne Zweifel mit den lokalen Verhältnissen im Rheindelta zusammenhängt.

Die Grenzen dieser Bezirke werden durch eine Konzentration der Arealgrenzen markiert. Die ansehnlichen täglichen Salzgehaltsschwankungen formen eine effektive Barriere sowohl für viele euryhaline marine Arten, als auch für fast alle Süßwasserorganismen. Der enorme Effekt dieser Salzgehaltsschwankungen wird deutlich demonstriert in Tabelle 1, in welcher die niedere Salinitätsgrenze einiger euryhaliner mariner Organismen in den niederländischen Aestuaren im Vergleich mit den äußersten Grenzen derselben Arten in der viel stabileren Ostsee (SEgERSTRÅLe 1957, H. LutHer 1950, A. LUTHER 1960, WAERN 1952) angegeben sind.

Nur ein verhältnismäßig kleiner Teil der Arten hat ungefähr dieselben niederen Salzgehaltsgrenzen in beiden Gebieten, z. B. Fucus vesiculosus L. und eine Reihe von Kleinturbellarien. Für die anderen Arten verursachen die fortwährenden täglichen Salzgehaltsschwankungen eine $\mathrm{zu}$ große Belastung ihres osmoregulatorischen Apparates und verhindern daher ihre Ansiedlung. Andere Faktoren als der Salzgehalt können auch regionale Differenzen in den äußersten Salzgehaltsgrenzen bewerkstelligen (REMANE 1959). Bei Blidingia minima (NÄG.) KYLIN und Nereis diversicolor (O. F. MüLLER) ist in der Ostsee die Temperatur vielleicht ein entscheidender Faktor.

Nicht allein in Aestuaren, sondern auch in anderen kontinuierlichen Ubergängen zwischen Meer und Süßwasser, wie der Ostsee, zeigt sich eine Differenzierung in einen oligohalinen, einen mesohalinen und einen polyhalinen Bezirk, entsprechend der Zusammensetzung der Flora und Fauna. Es ist aber zweifelhaft, ob wir die entsprechenden Bezirke in der Ostsee und den Aestuaren als gleichwertige Einheiten miteinander vergleichen dürfen. Das „osmotische Klima“ ist in beiden Gebieten äußerst verschieden. In den Aestuaren werden die Arealgrenzen tatsächlich bestimmt durch das mehr oder weniger entwickelte Vermögen der Arten, sich den großen Salzgehaltsschwankungen anzupassen. In der Ostsee sind die Grenzen, soweit sie von Salzgehaltsverhältnissen bestimmt sind, mehr eine Funktion der absoluten Salztoleranz der betreffenden Arten. Viele Arten, welche in den Aestuaren auf das Polyhalinikum beschränkt sind, besiedeln in der Ostsee auch das Mesohalinikum und eine kleine Zahl dringt selbst in das Oligohalinikum vor.

\section{INNERHALB DER DEICHE GELEGENE BRACKWASSER}

Die Aufstellung eines Systems für die Biozönosen der brackigen Binnengewässer ist eine statistische Angelegenheit. Im Gegensatz zu den Aestuaren und Bradkmeeren handelt es sich hier nicht um ein Kontinuum, das wir durch die Anwendung von Konzentrationen der Arealgrenzen untereinteilen können. Im Gegenteil, jeder Wasser- 
körper ist eine ökologische Einheit, mit ihrem eigenen jährlichen Salinitätszyklus, ihren eigenen Maximal- und Minimalwerten, ihrem eigenen Temperaturverlauf, ihrem eigenen Sauerstoffhaushalt, und ihrer eigenen Flora und Fauna. Infolgedessen hat kein Tümpel dieselben Eigenschaften wie ein anderer.

Es ist nicht schwierig, die Biozönosen dieser Tümpel und Gräben mit dem mittleren Salzgehalt in Zusammenhang zu bringen, und eine Einteilung in 3 Typen ist in großen Zügen möglich:

1. Die oligohalinen Gewässer werden hauptsächlich besiedelt von Süßwasserorganismen und nur wenigen Brackwasserarten; sie haben einen mittleren Salzgehalt von 0,3 bis etwa $2 \% \mathrm{Cl}^{\prime}$.

2. Die mesohalinen Gewässer enthalten vorwiegend Brackwasserarten, aber auch noch mehrere euryhaline limnische Formen, während die euryhalinen marinen Arten zahlenmäßig nicht von Bedeutung sind; ihre mittlere Salinität beträgt etwa 2 bis 7 (8) $\% \mathrm{Cl}^{\prime}$.

3. Die polyhalinen Gewässer haben eine Flora und Fauna, welche aus vielen Brackwasserarten und zahlreichen euryhalinen Meeresarten zusammengesetzt sind; sie enthalten nur eine zu vernachlässigende Anzahl Süßwasserarten; ihre mittlere Salinität ist $7(8)$ bis etwa $15 \% \mathrm{Cl}^{\prime}$.

Natürlich können zwischen diesen drei Typen Ubergänge nachgewiesen werden.

In fast allen diesen Gewässern treten mehr oder weniger große Salzgehaltsschwankungen auf, und zwar unter dem Einfluß von Niederschlag, Verdunstung, durch die Deiche dringendem Meerwasser und Zufluß von Süß- oder Brackwasser über die Poldergräben. Die Zusammensetzung der Biozönosen wurde durch diese Schwankungen in dem Sinne beeinflußt, daß alle stenohalinen Arten fehlen und daß die extremen niederen oder oberen Salzwerte für bestimmte euryhaline Arten letal sein können. In diesem Zusammenhang sind die Dauer der Extremverhältnisse und die Jahreszeit, in welcher sie stattfinden, von großer Bedeutung. In den meist labilen Gewässern können sich jedes Jahr Extremverhältnisse ausbilden und demzufolge sind ihre Biozönosen artenarm. In anderen Gewässern, in denen extreme Salzgehaltswerte nur episodische Erscheinungen darstellen, können diese trotzdem die Abwesenheit von bestimmten Arten hervorrufen, welche man sonst auf Grund des mittleren Salzgehaltes und anderer Umweltverhältnisse in dem betreffenden Biotop sicher erwartet hätte.

Die Arten, die diese innerhalb der Deiche gelegenen Brackwässer besiedeln, sind selbstverständlich in hohem Maße euryhalin. Die folgenden Beispiele mögen das belegen.

Ruppia spiralis L. ex Dum. dominiert in Gewässern mit einem mittleren Salzgehalt von $8-15 \% \mathrm{Cl}^{\prime}$, sie ist jedoch noch ziemlich zahlreich vertreten in Gewässern mit niedrigeren mittleren Salinitäten, aber nicht unter $6 \% \mathrm{Cl}^{\prime}$. Diese Daten gelten unter der Bedingung, daß der Minimalsalzwert niemals unter 2\% $\mathrm{Cl}^{\prime}$ fällt. Der Maximalsalzgehalt, welchen die Art ertragen kann, ist außerordentlich hoch. Im Jahre 1959 überlebte sie in der Suzanna-inlaag (Schouwen) einen Maximalsalzwert von $64 \%$ $\mathrm{Cl}$. Unter diesen Umständen blühte die Pflanze nicht und machte einen dürftigen Eindruck.

Die Assel Idotea chelipes (Pallas) wurde in Gewässern mit einer mittleren Salinität von $8-15 \% \mathrm{Cl}^{\prime}$ gefunden, in denen die Minimalsalzwerte niemals unter $2 \%$ 
$\mathrm{Cl}^{\prime}$ fielen. Die obere Salinitätsgrenze dieser Art beträgt $\pm 30 \% \mathrm{Cl}^{\prime}$. In der Ostsee liegt die untere Salinitätsgrenze dieser Art bei $2,5 \% \mathrm{Cl}^{\prime}$.

Eine andere Assel, Sphaeroma bookeri LEACH, lebt in Brackwässern mit einer mittleren Salinität von 2,5-11\% $\mathrm{Cl}^{\prime}$, vorausgesetzt, daß die Minimalsalzwerte niemals unter $0,5 \% \mathrm{Cl}^{\prime}$ fallen und die Maximalwerte niemals $14 \% \mathrm{Cl}^{\prime}$ überschreiten. In der Ostsee liegt die untere Salzgrenze dieser Art bei 3,7\% $\mathrm{Cl}^{\prime}$ und ist vermutlich klimatologisch bedingt (Forsman 1952).

Obschon die drei genannten Arten große Salinitätsschwankungen gut vertragen können, fehlen sie ganz in den niederländischen Aestuaren, wo die Schwankungen von derselben Größenordnung sind wie in den Binnengewässern. Es gibt aber einen sehr wichtigen Unterschied zwischen beiden Typen. Der Salzgehalt wechselt in den Aestuaren während jedes Gezeitenzyklus zwischen einem Maximum und einem Minimum und umgekehrt, während sich in den brackigen Binnengewässern der komplette Salinitätszyklus im Verlaufe eines Jahres vollzieht. Andere Unterschiede - wie das Fehlen der Gezeitenströmungen und des Tidenhubes in den Binnengewässern - spielen nur eine sekundäre Rolle. Die genannten Arten können nur in Gezeitengewässern existieren, wenn der Tidenhub ziemlich gering ist.

Es kommt vor, daß mehrere Arten, welche in den Aestuaren deutlich begrenzte Salinitätsbezirke besiedeln, in den brackigen Binnengewässern mit Bezug auf die mittlere Salinität eine ganz andere Verbreitung zeigen. Einige dieser Fälle sind in Tabelle 2 angegeben.

Tabelle 2

Die mittleren Salinitätsbereiche (in \% $\mathrm{Cl}^{\prime}$ ) einiger Arten in den Aestuaren und brackigen Binnengewässern des südwestlichen Teiles der Niederlande. Extremwerte in Klammern.

\begin{tabular}{|lcc|}
\hline & Aestuare & Binnengewässer \\
\hline Gammarus duebeni LILlJEBorg & $0,3-7$ & $(0,1) 0,3-13(15,5)$ \\
Gammarus zaddachi SEXToN & Süßwasser-5 & $(0,5) 2,5-13(15,3)$ \\
Jaera marina ssp. ischiosetosa ForsmaN & \pm 3 & $(2,8) 4,8-15,2 *(33)$ \\
Ligia oceanica (L.) & $0,3->16,5$ & $8-15,2(64)$ \\
Potamopyrgus jenkinsi (SMITH) & Süßwasser-1,8 & Süßwasser-5(9) \\
* Der höchste bisher in holländischen Binnengewässern gefundene mittlere Wert
\end{tabular}

Die Tatsache, daß die Schnecke Potamopyrgus jenkinsi (Sмтт) in Binnengewässern höhere Salinitätsmittelwerte vertragen kann als in Aestuaren, ist eine unmittelbare Folge des milderen osmotischen Klimas in den erstgenannten Gewässern. Die Grenze der Amphipoden Gammarus duebeni Lulujeborg und G. zaddachi Sexton werden in den Aestuaren nicht in erster Linie durch die wechselnden Salzgehaltsverhältnisse bestimmt, sondern durch Konkurrenz mit verwandten Taxa (DEN HARTOG, 1964). Dasselbe gilt wohl für Jaera marina spp. ischiosetosa ForSman, welche in den aestuarinen Gewässern mit einer mittleren Salinität über $3 \% \mathrm{Cl}^{\prime}$ vollkommen durch J. m. ssp. syei BocQUET ersetzt ist. Im Falle von Ligia oceanica (L.) ist Konkurrenz mit schwach salztoleranten Landisopoden der grenzsetzende Faktor an den Ufern der Binnengewässer. An den Ufern der schwachsalzigen Teile von Aestuaren kann sich diese 
Art nur behaupten dank der episodisch auftretenden Überflutungen mit stark salzhaltigem Wasser bei Stürmen oder Springfluten.

Mehrere Untersucher haben auf die Wichtigkeit der Salinitätsschwankungen in brackigen Binnengewässern hingewiesen und haben versucht, diesen Faktor in dem Klassifizierungssystem zum Ausdruck zu bringen, z. B. Aguesse (1958) und D'ANCoNA (1959). Aber ihre Lösungen basieren nicht auf biologischen Unterschieden, sie sind vielmehr als nomenklatorische Kunstgriffe zu betrachten.

Aguesse (1958) klassifizierte die brackigen Binnengewässer in 4 Poikilohalinitätsklassen, doch als eine Folge ihrer Definitionen sind sie nicht unabhängig von einer Salinitätsklasseneinteilung. So ist ein Wasserkörper oligopoikilohalin, wenn die Maximal- und Minimalwerte in dieselbe Kategorie des Salinitätsklassensystems fallen. Wenn der Maximalsalzwert in die Kategorie unmittelbar über derjenigen in welcher das Minimum liegt fällt, spricht er von mesopoikilohalinem Wasser. Und wenn das Maximum in der Salinitätsklasse, welche zwei Kategorien über derjenigen, in welcher das Minimum liegt, gelegen ist, nennt er den betreffenden Wasserkörper polypoikilohalin. Es gibt mehrere Fälle von mesopoikilohalinem und polypoikilohalinem Wasser, je nachdem in welchex Kategorie der mittlere Salzwert gelegen ist. Die Gewässer, in welchen der Salzhaushalt durch menschliche Einflüsse, z. B. das Ein- und Abführen von Süß- oder Meerwasser durch Schleusen und Düker, verändert wird, werden von AgUesse als eine besondere sub poikilohaline Klasse betrachtet. Diesem sehr komplizierten und künstlichen System entspricht kein einziges biologisches Prinzip. Alle brackigen Binnengewässer in den Niederlanden, so verschieden sie auch sein mögen, müßten nach diesem System als subpoikilohalin betrachtet werden.

Die Lösung von D'ANCONA (1959) ist einfacher, da er - wenn die Maximal- und die Minimalsalzwerte in verschiedenen Salinitätsklassen gelegen sind - nur ihre Namen kombiniert. So spricht er z. B. von meso-polyhalinem Wasser oder von poly-hyperhalinem Wasser. Obschon diese Lösung frei ist vot unnötiger Kompliziertheit, basiert dieses System auf der Klassifizierung nach dem Salzgehalt und nicht nach biologischen Merkmalen.

Doch selbst, wenn man die Einteilung nach der Salinität akzeptiert, gibt das System ein sehr ungenaues Bild der Salzgehaltsschwankungen, und ich frage mich, ob es nicht fruchtbarer wäre, absolute Zahlen zu geben und daneben einen Instabilitätskoeffizienten einzuführen. Die Normalinstabilität kann definiert werden als der Quotient zwischen der mittleren jährlichen Salinitätsschwankung und der mittleren jährlichen Salinität, beide bestimmt während derselben Periode: Die absolute Instabilität ist der Quotient zwischen der maximalen Salinitätsschwankung und der mittleren jährlichen Salinität, beide während derselben Periode bestimmt. In dem absoluten Instabilitätskoeffizienten kommen auch temporäre und episodische Erscheinungen, wie die Wirkungen extrem trockener Sommer oder sehr regenreicher Winter, zu ihrem Recht. Die Bedeutung der Instabilität werde ich anhand mehrjähriger Beobachtungen in 19 innerhalb der Deiche gelegenen niederländischen Brackwässern in einer späteren Arbeit noch näher erläutern. 


\section{EIN TYPOLOGISCHES SYSTEM DER BRACKWASSER}

Wenn wir die vorher besprochenen Brackwassertypen miteinander vergleichen, zeigt es sich, daß jeder Typ an Hand der Komposition von Flora und Fauna unterteilt werden kann in einen oligohalinen, einen mesohalinen und einen polyhalinen Teil. Die Grenzen der entsprechenden Untertypen können nicht mit denselben mittleren Salinitätsgrenzen korreliert werden, und - was wichtiger ist - sie werden in jedem Typ durch verschiedene Faktoren bestimmt. Die Konsequenz hiervon ist, daß ein Klassifizierungssystem, das auf der Korrelation zwischen den Biozönosen und der mittleren Salinität basiert, niemals vollkommen zutrift. Wenn wir zu einem besser verwendbaren Einteilungsschema gelangen wollen, ist es notwendig, einen anderen Ausgangspunkt zu wählen.

Zwischen den beiden homoiohalinen Medien, dem Süßwasser und dem Meer, befindet sich eine Grenzzone, in der eine Mischung von Süß- und Meerwasser stattfindet: das Mixohalinikum oder das Brackwasser. Diese Übergangszone kann von großer Ausdehnung sein, mit einer nahezu kontinuierlichen allmählichen Anderung der Salinität, aber die Salzgehaltsumwandlung kann auch mehrere Diskontinuitäten (Sprungzonen, Sprungschichten) aufweisen. Der Übergang zwischen Meer- und Süßwasser kann auch sehr abrupt sein, und weiter gibt es Fälle, wo der Übergang zwischen Meerwasser und Süßwasser zeitweilig blockiert oder sehr erschwert wird.

Die Übergangszone muß betrachtet werden als ein fortwährendes Konfliktgebiet zwischen Meerwasser und Süßwasser. Periodisch dehnt das Meer seinen Einfluß aus und wird in der darauffolgenden Periode durch das Süßwasser wieder zurückgedrängt. Die Salinität verändert sich also nicht nur im Raum, sondern auch in der Zeit. Die Salinität des Brackwassers ist also unstabil im Gegensatz zu dem Salzgehalt des Meeres und des Süßwassers. Die Periodizität in den Salinitätsveränderungen wird verursacht durch die Gezeiten und durch die Abfuhr des Niederschlagsüberschusses (d. h. die Differenz zwischen dem Niederschlag und der Verdunstung), welche eine jährliche Periode aufweist.

Die verschiedenen Konfliktsituationen, welche bei dem Übergang vom Süßwasser zum Meer auftreten können, sind sehr gut anhand ihrer Kontinuität und der Periodizität der Salzgehaltsschwankungen in einem typologischen System zu klassifizieren. Ich habe 8 Typen unterschieden:

\section{A. Kontinuierlicher Übergang zwischen Meer und Süßwasser}

1. Ein sehr allmählicher Übergang mit sehr geringen jährlichen Salinitätsschwankungen kommt in der Natur nur in den ausgedehnten Brackmeeren vor, z. B. in der Ostsee und dem Schwarzen Meer.

2. Ein kontinuierlicher Ubergang mit ansehnlichen Salinitätsschwankungen wird in Flußmündungen in einem gezeitenlosen Meer angetroffen. Die Normalsituation kann zeitweilig gestört sein, z. B. durch Stürme oder andere episodisch auftretende Verhältnisse. 
3. Dasselbe gilt für Aestuare, aber hier treten unter dem Einfluß der Gezeiten auch $12^{1 / 2}$-stündliche Salinitätsschwankungen auf. Weiter zeigt der Salinitätsgradient hier oft Diskontinuitäten, welche jedoch nicht permanent sind.

\section{B. Abrupter Übergang zwischen Meer und Süßwasser}

4. Kleine Süßwasserströmchen, welche ins Meer münden, unterliegen in der Gezeitenzone während jedes Gezeitenzyklus zweimal einem plötzlichen Salinitätssprung, von einem Minimum zu einem Maximum und umgekehrt. Diese Salzsprünge vollziehen sich in wenigen Augenblicken. Zwischen diesem "Schockbiotop" und den Aestuaren bestehen Übergänge. Der wichtigste Unterschied ist, daß in dem Schockbiotop die Menge des Süßwassers zu vernachlässigen ist, sobald es sich mit dem Meerwasser mischt.

\section{Zeitweilige oder semipermanente Blockierung des Übergangs zwischen Meer und Süßwasser}

5. Supralittorale Tümpel haben unter dem Einfluß der Springtiden einen 14- oder 28-tägigen Salinitätszyklus. In diesen Gewässern wird der Salzgehalt weitgehend bestimmt durch Niederschlag, Verdunstung und salziges Spritzwasser, aber während jeder periodischen oder episodischen (Stürme!) Überflutung wieder zurückgebracht auf das marine Salinitätsniveau. Im Sommer werden viele Tümpel aber nicht vom Meer erreicht und es ist dann vollkommen abhängig von dem Klima, ob sie in dieser Zeit einen Süßwasser- oder einen hyperhalinen Charakter annehmen.

6. Es gibt viele Gewässer, in denen der direkte Einfluß des Meeres weitgehend blokkiert oder reguliert wird, z. B. in den Lagunen, den „Étangs“" und den innerhalb der Deiche gelegenen Brackwässern. Der jährliche Salinitätszyklus ist selbst in nebeneinander gelegenen Gewässern sehr verschieden, da nicht nur die Zufuhr von Salzwasser und die klimatischen Verhältnisse die entscheidenden Faktoren sind, sondern auch das Vernältnis zwischen Wasservolumen und Wasseroberfläche eine wichtige Rolle spielt. In seichten Tümpeln steigt die Salinität während trockener Sommer weit über die des Meeres an.

\section{Andere Fälle}

7. Die Gezeitenzone, in welcher die marine Flora und Fauna während des Trockenfallens direkt dem Niederschlag und der Verdunstung ausgesetzt sind, ist nach DAHL (1959) tatsächlich ein poikilohalines Milieu.

8. Das Küstengrundwasser (REMAnE 1958).

Diese Brackwassertypen zeigen natürlich viele andere Unterschiede, z. B. im Temperaturverlauf, Sauerstoffhaushalt, Vorkommen von Schwefelwasserstoff, Wasser- 
strömung, usw. REMANE (1959) hat diese Besonderheiten für die Brackmeere, die Aestuare und die Lagunen dargestellt. Die verschiedenen Typen sind biologisch durch treue Leitarten charakterisiert; es gibt nämlich Arten, welche nur auf einen oder einige dieser Typen beschränkt sind. Ich werde hier die Zahl der Beispiele begrenzen; Die Schnecke Assiminea grayana Fleming kommt nur in Aestuaren vor und die Amphipoden $\mathrm{Ma}$ rinogammarus stoerensis (ReID) und $M$. pirloti SEXTON \& SPooner sowie der Plattwurm Procerodes littoralis (STRÖM) sind in hohem Maße charakteristisch für den Schockbiotop; die supralittoralen Tümpel werden gekennzeichnet durch sehr spezielle Kleinalgen, wie Brachiomonas-, Platymonas- und Asteromonas-Arten und den Copepoden Tigriopus fulvus (FISCHER).

In mehreren dieser Brackwassertypen, z. B. in den kontinuierlichen Brackwässern, aber auch in den blockierten lagunären Gewässern ist eine Dreiteilung nach der $\mathrm{Zu}$ sammensetzung der Biozönosen möglich. Wie schon oben betont wurde, können die Grenzen der entsprechenden Biozönosen in den verschiedenen Typen nicht mit denselben Salzgehaltswerten korreliert werden, so daß die Korrelation mit der mittleren Salinität in jedem Typ gesondert bestimmt werden muß.

\section{ZUSAMMENFASSUNG}

1. Wenn das „Venice System“ für die Klassifizierung der Brackwässer auf biologischen Unterschieden beruht, sollten die verschiedenen Klassen nicht in erster Stelle nach der Salinität, sondern nach den biologischen Erscheinungen definiert werden.

2. Auf Grund der Zusammensetzung der Biozönosen ist es möglich, in Brackmeeren, Aestuaren und abgeschlossenen Brackwässern drei Untertypen zu unterscheiden, deren Grenzen mit dem mittleren Salzgehalt korreliert werden können, und welche gewöhnlich als oligohalin, mesohalin und polyhalin bezeichnet werden.

3. Die entsprechenden Untertypen der drei verschiedenen Brackwassertypen sind aber nicht vergleichbar, da ihre Grenzen durch ganz verschiedene Faktoren bestimmt werden und nicht zusammenfallen; infolgedessen weisen ihre Biozönosen deutliche Differenzen auf.

4. Die großen täglichen Salzgehaltsschwankungen in den Aestuaren formen eine effektive Barriere für viele euryhaline Meeresarten und fast alle Süßwasserarten.

5. In den abgeschlossenen Brackwässern verursachen die oft großen jährlichen Salinitätsschwankungen das Fehlen stenohaliner Arten. Die Maximum- und Minimumsalzwerte entscheiden, welche euryhalinen Arten in einem bestimmten Wasser gut oder nicht existieren können, und zwar unabhängig vom Schwankungsmuster.

6. Eine Einteilung des Brackwassers nach seiner räumlichen Kontinuität und der Periodizität dex Salzgehaltsschwankungen ergibt ein typologisches System, in welchem folgende Typen erkannt wurden: a) Brackmeere; b) Flußmündungen in einem gezeitenlosen Meer; c) Aestuare; d) Schockbiotope; e) supralittorale Tümpel; f) lagunäre und abgeschlossene Brackwässer; g) Gezeitenzonen; h) Küstengrundwasser. Die verschiedenen Typen werden von speziellen Leitarten gekennzeichnet.

7. Nur ein Teil dieser Brackwassertypen zeigt eine weitere Unterteilung, welche mit dem mittleren Salzgehalt korreliert werden kann. 


\section{ZITIERTE LITERATUR}

Aguesse, P., 1958. La classification des eaux poikilohalines, sa difficulté en Camargue; nouvelle tentative de classification. Vie et Milieu 8, 341-365.

D'Ancona, U., 1959. The classification of brackish waters with reference to the north Adriatic lagoons. Archo Oceanogr. Limnol. 11 Suppl., 93-109.

Caspers, H., 1959. Die Einteilung der Bradkwasser-Regionen in einem Aestuar. Archo Oceanogr. Limnol. 11 Suppl., 153-169.

DaHL, E., 1959. Intertidal ecology in the terms of poikilohalinity. Archo Oceanogr. Limnol. 11 Suppl., 227-237.

Forsman, B., 1952. Uber das Vorkommen der Gattung Sphaeroma in nordischen Gewässern. Ark. Zool. 4, 153-158.

Hartog, C. DEN, 1960. Comments on the Venice-system for the classification of brackish waters. Int. Rev. Hydrobiol. 45, 481-485.

- 1961. Die faunistische Gliederung im südwestniederländischen Deltagebiet. Int. Rev. Hydrobiol. 46, 407-418.

- 1964. The amphipods of the deltaic region of the rivers Rhine, Meuse and Scheldt in relation to the hydrography of the area. Pt 3. Neth. J. Sea Res. 2, 407-457.

Luther, A., 1960. Die Turbellarien Ostfennoskandiens I. Acoela, Catenulida, Macrostomida, Lecithoepitheliata, Prolecithophora und Proseriata. Fauna fenn. 7, 1-155.

Luther, H., 1950. Die Funde von Zostera marina L. in der nördlichen Ostsee. Memor. Soc. Fauna Flor. fenn. 25, 25-36.

Petit, G. \& Schachter, D., 1959. Les étangs et lagunes du littoral méditerranéen français et le problème de la classification des eaux saumâtres. Archo Oceanogr. Limnol. 11 Suppl., 75-91.

REDEKE, H. C, 1933. Über den jetzigen Stand unserer Kenntnisse der Flora und Fauna des Brackwassers. Verh. int. Ver. Limnol. 6, 46-61.

Remane, A., 1959. Regionale Verschiedenheiten der Lebewesen gegenüber dem Salzgehalt und ihre Bedeutung für die Brackwasser-Einteilung. Archo Oceanogr. Limnol. 11 Suppl., 35-46.

- \& SChlieper, C., 1958. Die Biologie des Brackwassers. In: Die Binnengewässer. Bd 22. Schweizerbart, Stuttgart, $348 \mathrm{pp}$.

Segerstråle, S. G., 1957. Baltic Sea. In: Treatise on marine ecology and paleoecology I. Ed. by J. W. Hedgpeth. Mem. geol. Soc. Am. 67, Vol. 1, 751-800.

- 1958. A quarter century of brackishwater research. Verh. int. Ver. Limnol. 13, 646-671.

- 1959. Bradkishwater classification, a historical survey. Archo Oceanogr. Limnol. 11 Suppl., 7-33.

VäLikangas, I., 1933. Über die Biologie der Ostsee als Brackwassergebiet. Verh. int. Ver. Limnol. 6, 62-112.

WaERn, M., 1952. Rocky-shore algae in the Oregrund Archipelago. Acta phytogeogr. suec. 30, $1-298$.

Final resolution of the symposium on the classification of brackish waters, 1959. Archo Oceanogr. Limnol. 11 Suppl,, 243-245.

\section{Diskussion im Anscbluß an den Vortrag DEN HARTOG}

REMANE: Im großen ganzen stimmen Thre Untersuchungsergebnisse recht gut überein mit den Vorstellungen, die wir bisher hatten. Sie kritisieren das System Venedig. Ich war selbst dabei, und es ging bei der Schlußformulierung ebenso zu wie bei vielen politischen Kongressen. Es war ein Kompromiß, und zwar standen sich in Venedig zwei Gruppen gegenüber: eine, die auf den Salzgehaltswechsel und die andere, die auf den Salzgehalt an sich besonderes Gewicht legte. Man kann natürlich von beiden Seiten ausgehen, und in der Natur wirken beide Faktoren kombiniert. In der Ostsee, wie Sie ja auch betont haben, können wir große Gebiete Brackwassers haben mit minimalem Salzgehaltswechsel. Dort können wir die Beziehungen zum Salz- 
gehalt an sich darstellen. Astuare und Lagunen, auf der anderen Seite, sind Gebiete extremen Salzgehaltswechsels. Nun kann man anfangen mit dem Salzgehalt an sich und fragen, welche Korrektionen müssen wir an den Beziehungen anbringen, wenn Salzgehaltswechsel da ist. Die Korrektionen sind klar: Salzgehaltswechsel kann als zusätzlicher, hindernder Faktor auftreten, daß also die marinen Organismen eher aufhören und auch die limnischen, als es in einem gleichmäßigen Niveau der Fall ist. Kurzlebige Organismen, wie Rotatorien etc., die inaktive Dauerstadien haben, können im Wechselmilieu die Salzgehaltsgrenzen weiter hínausschieben, wenn nur eine kurze Periode ihrem Salzgehaltsbedürfnis entspricht. Die anderen Zeiten uberdauern sie eben in einem inaktiven Zustand. Ich glaube, wenn man vom Salzgehalt ausgeht und die Korrektion anbringt, kommt man ziemlich mit dem überein, was Sie gefunden haben. Dabei ist nicht der Biotop-Faktor zu vergessen. In Astuaren verschwinden Biotope, die im Brandungsgebiet natürlich da sind, und wenn Idotea granuloset in der Ostsee so viel weiter geht als bei Ihnen und auch bei uns in Strandgebieten, so beruht das darauf, daß sie bewegtes Wrasser fordert, und das ist in der Ostsee bis zu niedrigen Salzgehalten gegeben, aber in diesem Sinne nicht in Astuaren. Ich glaube, bei Coelogynopora schulzii, die Sie ja bei einem sehr viel höheren Salzgehalt aufhören ließen als in der Ostsee, spielt vor allem der Biotop-Faktor eine Rolle. Haben Sie die holländischen Salzwiesen auf diese Art hin untersucht?

DEN HARTOG: Ich habe die Marschen im südwest-holländischen Deltagebiet auf Coelogynopora scbulzii hin untersucht und diese Art in den euhalinen und polyhalinen Salzwiesen wiederholt gefunden. In mesohalinen Salzwiesen mit derselben Biotop-Struktur dagegen habe ich nur einmal ein steriles Exemplar gefunden. Ich kann daher leider nicht sagen, ob es sich dabei um dieselbe Art handelt. Im Küstengrundwasser wurden keine Untersuchungen durchgeführt. Bezüglich einer ausführlichen Beschreibung der Okologie dieser Art in den holländischen $\mathrm{Ge}$ wässern verweise ich auf meine Arbeit in Proc. Kon. Ned. Akad. Wet. C 67, 29-33, 1964.

KinNe: Der Versudh von Herrn den Hartog, das sogenannte System von Venedig auf eine breitere Grundlage zu stellen, ist zu begrüßen. Ich möchte meinen, daß insbesondere die Einbeziehung periodischer und unperiodischer Salzgehalts $\mathrm{s} \mathrm{ch}$ w a $\mathrm{k}$ u $\mathrm{ng}$ e $\mathrm{n}$ in die Betrachtung einen wichtigen Vorteil darstellen. Auf jeden Fall hat er sich keine leichte Aufgabe gestellt. Vorkommen und Abundanz eines Organismus hängen - außer von dessen eigenen Beschaffenheit -- in der Regel von einer Vielzahl abiotischer und biotischer Faktoren und deren Fluktuationsmustern $a b$. Gegenwärtig verfügen wir weder über die technischen Möglichkeiten, alle beteiligten Umweltqualitäten zu messen, noch über ausreichende Kenntnisse, um deren Einflüsse auf lebende Systeme in allen Fällen hinreichend zu beurteilen. Es ist daher erforderlich, daß wir uns zunädhst auf sogenannte "Meisterfaktoren" beschränken. Eine solche Beschränkung darf aber nicht zu weit gehen. In der Regel lassen sich Verbreitung und Abundanz nur in Spezialsituationen auf die Intensitätsveränderungen e ines e inzigen Umweltfaktors zurückführen. Wenn es sich bei diesem einen Faktor dazu noch um den Salzgehalt handelt, wird die Situation besonders kritisch. Denn: Salzgehalt ist definiert als die Gesamtmenge an gelösten Stoffen pro Kilogramm Meerwasser unter der Annahme, daß alle Karbonate in Oxyde, die Bromide und Jodide in Chloride überführt und die gesamte organische Substanz oxydiert ist. Schon aus dieser Definition geht hervor, daß der Begriff Salzgehalt mehrere Aspekte umfaßt, denen eine unterschiedliche biologische und physiologische Bedeutung zukommt. So kann der Faktor Salzgehalt lebende Systeme beeinflussen durch Veränderungen der Gesamtosmokonzentration, der relativen Anteile gelöster Komponenten, der Absorptions- und Sättigungskoeffizienten für gelöste Gase (insbesondere $\mathrm{O}_{2}$ und $\mathrm{CO}_{2}$ ), der Dichte und Viskosität sowie der Strahlungsabsorption, Schallïbertragung, elektrischen Leitfähigkeit etc. Die meisten dieser Aspekte sind ihrerseits eine Funktion der Temperatur. Wenn man also ein System aufstellen will, das über eine örcliche Bedeutung hinaus Anspruch auf allgemeinere Gültigkeit erheben kann, dann sollte man außer dem Salzgehaltsfaktor zumindest den Temperaturfaktor berücksichtigen und gegebenenfalls auch das Lichtklima einbeziehen. Letzteres vor allem im Hinblick auf die Primärproduktion und vertikale Verbreitungsgradienten.

LÜNEBURG: Ich darf vielleicht zu den Ausführungen von Professor KinNe noch hinzufügen, daß ja auch die physikalischen Verhältnisse als solche ein auf einen einzigen Faktor zuge- 
schnittenes System stören würden. Denken wir einmal an die starke Trübung, die ja häufig vor allem im Bereich der Astuare vorhanden ist. In den Trübungswolken haben wir einen Extinktionsschwund, der 2 bis 3 Zehnerpotenzen beträgt. Auch als Nicht-Biologe könnte ich mir vorstellen, daß derartige Veränderungen floristisch und faunistisch von Bedeutung sind.

Remane: Hier sind zwei Möglidkeiten der Okologie aufgetreten. Das Tier lebt in einer Umwelt, und Umwelt ist ein ganz komplexes chemisches und physikalisches und biotisches System. Der Okologe sieht sich einem unfaßbaren System gegenüber. Er darf aber nicht kapitulieren, sondern er wird erstens einmal anfangen und je nach der Situation mit einem Faktor beginnen und dann die Beeinflussungen weiterer Faktoren sekundär addieren. Zunächst wird man sich einen Ort suchen, wo nur der eine Faktor variiert und die übrigen Faktoren möglichst konstant sind. Man wird also ein Schema erarbeiten und es dann später durch Zusatzfaktoren, wie kurzfristigen oder langfristigen Wasserwechsel, modifizieren. Dadurch erhält man natürlich sehr verschiedene Ortsmuster. Man kann aber diese verschiedenen Muster als sekundäre Komm plikationen des Ausgangsschemas betrachten. Sie gelten dann nur ungefähr für den ideal gedachten Lebensraum, der nur in einem Faktor variiert und in den anderen konstant ist. Solche idealen Situationen können wir selbst im Experiment nur sehr schwer herstellen, weil immer wieder Faktoren hinzutreten, die man beim Experiment zunächst nicht beachtet hat, die dann aber sekundär wichtig erscheinen. Insofern sind audn experimentell untermauerte Grundsysteme mit Unsicherheitsfaktoren und mit Modifikationsnotwendigkeiten ausgestattet. Aber wir haben ja die Aufgabe, die Modifikation - das ist hier überzeugend herausgekommen - für den jeweiligen Ort herauszuarbeiten. Es war mir aber auf der anderen Seite ein tiefer Eindruck, als ich einmal in Südbrasilien Brackwassergebiete besah und auf einmal eine ganze Reihe von Formen wiederfand, die ich von der Ostsee her kannte. Und genau so passierte das sofort im Schwarzen Meer und in anderen Gebieten. Es ist aber schon so etwas an dem Salzfaktor an sich wie an dem Temperaturfaktor für die Verteilung der Landorganismen oder wie an dem Niederschlagsfaktor für die Verteilung der Pflanzen.

Krnne (zu REMANE): Ich stimme Thnen voll und ganz darin $z u$, daß wir uns angesichts der Komplexität der $z \mathfrak{u}$ analysierenden Beziehungen von einfachen $z u$ komplizierteren Situationen. vorarbeiten müssen - daß wir also mit e in e $\mathrm{m}$ Faktor anfangen und dann einen zweiten oder dritten hinzunehmen. Auch darin, daß unsere Laboratoriumsexperimente ihre Sdhwächen haben, stimme ich mit Thnen iberein. Es ist daher notwendig, Untersuchungen in der freien Natur und im Laboratoriumsexperiment parallel nebeneinander zu betreiben. Bedenken habe ich aber hinsichtlich eines Sys te m s, welches, wie das Venedig-System, auf einem einzigen ökologischen Faktor basiert. Allen hier vorgebrachten Einschränkungen zum Trotz beinhaltet der Begriff "System" einen gewissen Anspruch auf allgemeinere Gültigkeit. Ein einmal akzeptiertes System führt leicht zu einer Einschränkung der Flexibilität bei der Beurteilung neuer Ergebnisse und fördert die Einrangierung neuer Informationen auf vorher verlegten Gleisen. Daß der Salzgehalt in zahlreichen Brackgewässern - wie etwa der Ostsee mit ihrem ausgeprägten West-Ost-Gradienten - einen ganz erheblichen Einfluß auf die Verbreitung und Vermehrungsrate besitzen kann und einen "ökologischen Meisterfaktor" ersten Ranges darstellt, daran besteht gar kein Zweifel. Ebenso sicher ist der Salzgehalt als ökologischer Faktor aber im marinen Bereich von untergeordneter Bedeutung und im offenen Ozean möglicherweise ganz zu vernachlässigen. Ich wollte hier vor allem vor einer einseitigen Betrachtungsweise warnen und darauf hinweisen, daß für eine objektivere und weniger lokal bezogene Beurteilung der Situation die Einbeziehung der Temperatur und gegebenenfalls auch anderer wichtiger Umweltqualitäten erforderlich ist.

DEN HaRtog: Auch ich bin der Ansidht, daß mehrere Faktoren berücksidhtigt werden sollten. Das System, welches ich hier vorgeschlagen habe, beruht nicht nur auf einem Faktor. Ich habe nicht den mittleren Salzgehalt, sondern die periodischen und episodischen Salzgehaltsschwankungen berücksichtigt. Neben der Periodizität der Salinitätsfluktuationen habe ich die Kontinuität des Uberganges zwischen Meer und Süßwasser als ein wichtiges Einteilungskriterium hoch gewertet. Auf diese Weise kann eine Anzahl Brackwassertypen definiert werden, welche für jeden erkennbar sind. Mehrere Typen zeigen eine Dreiteilung hinsichtlich der Differenzierung der Flora und Fauna, welche mit dem mittleren Salzgehalt korreliert werden kann. 
Ich habe aber ausdrücklich betont, daß die Salzgehaltskorrelationen in jedem Typ gesondert bestimmt werden müssen, da jeweils ein anderer Faktor entscheidend für die Differenzierung sein kann. Da auch die Temperatur, regional betrachtet, ein Hauptfaktor ist - wie Herr KINNE soeben ausgeführt hat - ist tatsächlich eine Bestimmung der Salinitätsgrenzen der Flora und Fauna für jedes Wassersystem notwendig und letztlich nur von lokaler Bedeutung. Leider ist der Einfluß der Temperatur auf die biologische Gliederung in natürlichen Brackwässern noch nicht genügend bekannt, so daß dieser Faktor noch nicht in ein System für Brackwässer einbezogen werden kann. Die hier vorgeschlagene Typeneinteilung darf nicht betrachtet werden als eine erweiterte Form des "Venice Systems", da die Einteilungskriterien verschieden sind.

Koch: Zur Unterstiitzung des Laboratoriumsexperiments: Die von Ihnen genannte Bangia fuscopurpurea ist eine marine Art. Die im Süßwasser vorkommende Rotalge ist Bangia atropurpurea. Es gibt in der Ostsee noch Bangia pumila, die vielleicht - physiologisch gesehen zwischen ihnen steht. Die Monosporen von Bangia atropurpurea keimen nur in Salinitäten von 1,5 bis $10 \%$, die von Bangia fuscopurpurea nur im marinen Bereich um $32 \%$. Das angebliche Vorkommen von Bangia fuscopurpurea im Süßwasser bedarf also immer eines experimentellen Nachweises.

DEN HARTOG: Bangia fuscopurpurea ist eine marine Art, welche weit ins Brackwasser vordringt und gelegentlich wohl auch ins Süßwasser, vorausgesetzt, daß das Süßwasser eutroph ist oder stark verschmutzt. In der ehemaligen Zuiderzee, welche eine Salinität von 8 bis $10 \% \mathrm{Cl}^{\prime}$ hatte, war die Art nicht häufig, aber nach der Absperrung im Jahre 1932 und der nachfolgenden Aussüßung hat $B$. fuscopurpurea sich sehr ausgebreitet und wurde eine der dominanten Arten. Das gleiche passierte im Brielschen Meer, welches 1950 abgesperrt wurde. $B$. atropurpurea habe ich nur in sauberen oligo-mesotrophen Fließgewässern gefunden.

Kосн: Das Vorkommen von Bangia atropurpurea in ausgesprochen sauberen Gewässern und zugleich in stark eutrophierten ist auf das spezielle physiologische Verhalten dieser Alge zurückzuführen. 